\title{
Diferenciação como estratégia: um estudo de caso da Apple
}

\author{
Differentiation as strategy: an Apple case study
}

Recebido: 20/10/2021 | Revisado: 29/10/2021 | Aceito: 08/11/2021 | Publicado: 11/11/2021

\author{
Isabelly Borborema Gomes \\ ORCID: https://orcid.org/0000-0003-4057-7819 \\ Universidade Federal de Campina Grande, Brasil \\ E-mail: isabelly.gomes.8@gmail.com \\ Isabel Lausanne Fontgalland \\ ORCID: https://orcid.org/0000-0002-0087-2840 \\ Universidade Federal de Campina Grande, Brasil \\ E-mail: isabelfontgalland@gmail.com
}

\begin{abstract}
Resumo
A Apple Inc. é uma das maiores empresas de tecnologia de nosso tempo e responsável por diversos produtos que insurgiram nas relações contemporâneas sociedade e tecnologia de informação digital. Com foco em uma estratégia de inovação tecnológica, constante e forte diferenciação de produtos, a Apple investe de maneira expressiva em Pesquisa e Desenvolvimento (P\&D), tendo por diferencial competitivo a inovação em tecnologia de informação (T.I). O presente trabalho tem por objetivo geral entender e enquadrar a estratégia comercial da Apple como uma estratégia defensiva, através da MEC- metodologia de estudo de caso, onde se delineou o entendimento de toda a discussão em tela. Como resultados é imprescindível assinalar a inversão massiva em novos produtos e processos, serviços após venda e desenvolvimento de matriz suprimentos, alavancados por mudanças de não mais de dois anos.
\end{abstract}

Palavras-chave: Apple; Diferenciação; Tecnologia.

\begin{abstract}
Apple Inc. is one of the largest technology companies of our time and responsible for several products that have emerged in contemporary relationships between society and digital information technology. Focused on a strategy of technological innovation, constant and strong product differentiation, Apple invests significantly in Research and Development (R\&D), having innovation in information technology (IT) as a competitive advantage. The present work has as general objective to understand and frame Apple's commercial strategy as a defensive strategy, through the MECcase study methodology, which outlined the understanding of the entire discussion on screen. As a result, it is essential to point out the massive investment in new products and processes, after-sales services and development of the supply matrix, leveraged by changes of no more than two years.
\end{abstract}

Keywords: Apple; Differentiation; Technology.

\section{Introdução}

A criação de computadores e máquinas capazes de realizar operações e tarefas caracteriza uma das maiores evoluções no desenvolvimento tecnológico da humanidade. Brookshear (2013) nota que, por muitas décadas, os computadores eram restritos ao uso em universidades e agências governamentais. Devido às condições materiais e de conhecimento da época, os computadores eram gigantes, pesavam toneladas e não eram de fácil utilização, podendo citar como exemplo o Harvard Mark I, que pesava em torno de $4.500 \mathrm{~kg}$ e utilizava manivelas.

Diante de situações como essas, a Apple Inc. inovou a relação entre o cidadão comum e os computadores, principalmente através da criação do Apple II, conforme aponta Kahney (2008). Essa inovação, todavia, não parou por aí. Entre outros produtos revolucionários, lançados pela Apple, pode-se citar o iPod (2001), o iPhone (2007), além de aplicativos como o iTunes (2001), permitindo que se perceba que a inovação tecnológica e a diferenciação de produtos constituem dois dos pilares inseparáveis da vida moderna contemporânea. 


\section{Metodologia}

Segundo Lakatos e Marconi (2017), um método se caracteriza por um conjunto de atividades racionais que permite, com maior economia e facilidade, produzir conhecimentos válidos e verdadeiros. Nesse sentido, para este trabalho foi utilizado o método de estudo de caso que conforme aponta Yin (2001), é baseado em fontes de evidências variadas sobre eventos reais e conta com proposições teóricas anteriormente desenvolvidas, que auxiliarão no processo de coleta e análise de dados, permitindo aprofundar os conhecimentos sobre tais proposições ao observá-las na prática.

O estudo de caso tem em foco a empresa Apple, permitiu verificar, através da análise de alguns dos seus produtos lançados anteriormente - o Apple II e o iPod, o tipo de estratégia adotada pela empresa, especialmente no que diz respeito à inovação tecnológica e diferenciação de produtos, tendo na última o entendimento de melhoramento de processo de impulsão de mix de produtos.

\section{Resultados e Discussão}

\subsection{A Apple}

Em abril de 1976, Steve Jobs e Steve Wozniak fundavam a Apple Computers, Inc., nos Estados Unidos, mudando drasticamente o futuro da indústria de computadores com o lançamento do Apple I e, posteriormente, do Apple II, sendo este último um grande passo dado pela companhia, permitindo que consumidores que não tinham conhecimentos de informática avançados pudessem utilizá-lo. Os dois Steves tinham em mente, um mundo tecnológico acelerado e fácil de ser carreado em mãos. Daí veio o conceito de computer lap-top, que possibilita o consumidor de portar de forma convivial um instrumento de T.I.

Até o final dos anos 90, a empresa se ateve à produzir apenas computadores. Apostando, assim em T.I, design e na apresentação de software próprio. Apesar disso, a Apple enfrentou diversas dificuldades financeiras que a enfraqueceram no mercado, pois, além das dificuldades financeiras enfrentadas, perdeu espaço para a concorrência e seus produtos já não causavam o mesmo impacto. É a partir do retorno de Steve Jobs (que havia saído da empresa anteriormente) que cria-se uma mudança na estratégia de negócio, e de novo a Apple volta a ganhar espaço de mercado, ao introduzir o iMac e, posteriormente, um dos grandes sucessos dos anos 2000: o iPod.

Muitos anos se passaram desde então, sendo possível observar o admirável crescimento da empresa, que alcançou posição de liderança frente às empresas do mesmo segmento tecnológico. Não é por acaso que, em 2021, ela tenha ficado na $6^{a}$ posição entre as maiores empresas de capital aberto do mundo, de acordo com a Forbes (2021), e esse sucesso se deve ao fato de que a Apple, enquanto empresa, deixou de ser apenas uma fabricante de computadores e passou a competir em outros mercados, como o da telefonia móvel celular. Ao introduzir a primeira versão do iPhone, em 2007, se torna o maior standard de produto da linha de integração de mídia móvel com telefonia. Possibilitando assim vários dispositivos de softwares usando em compatibilidade com o telefone móvel, hoje conhecidos como smart-phones.

\subsection{Conceitos sobre inovação}

Conforme aponta Hasenclever e Ferreira (2013), a inovação tecnológica depende de um processo de investimento em P\&D, capaz de gerar novos conhecimentos úteis aplicáveis a produtos ou serviços que a empresa produz. Quando isso ocorre, há uma mudança tecnológica ou inovação.

Esse ciclo de inovação possui três estágios: invenção, inovação e imitação ou difusão. A invenção é a parte do ciclo de inovação, conforme aponta Hasenclever e Ferreira (2013), que cria produtos novos através de conhecimentos novos ou da organização de conhecimentos já existentes de uma maneira nova. É fruto, principalmente, do processo de P\&D, mas também 
da experiência particular da empresa e de seu ciclo de desenvolvimento e evolução. A invenção só se torna uma inovação, efetivamente, quando é lançada no mercado com sucesso comercial. Esse lançamento permite, por fim, um terceiro processo, que se chama imitação ou difusão, onde outras empresas tentam copiar, com maior ou menor grau de perfeição, a inovação.

Geralmente, a estratégia comercial de uma empresa em relação à inovação possui dois modos básicos: ofensiva ou defensiva. Conforme aponta Freeman e Soete (2008), a estratégia ofensiva é caracterizada pela empresa que lança novos produtos no mercado, sem qualquer relação com um produto anterior. São, digamos assim, "os primeiros", de tal modo que encaram todo o risco da inovação. A inovação é, aqui, uma inovação radical. Uma estratégia defensiva, ou imitativa, é caracterizada por altos investimentos em P\&D com vistas de imitar produtos novos lançados e, a partir de seus erros, melhorá-los, visando uma posição de destaque no mercado. Essa estratégia é a que mais se aproxima da utilização da diferenciação de produtos, conforme apontado por Freeman e Soete (2008) e Hasenclever e Ferreira (2013). Nesse caso, a inovação tem caráter incremental.

\subsection{A Apple e o Apple II}

Atualmente, é impossível imaginar um mundo em que não existam os computadores pessoais. Eles nos auxiliam em grande parte de nossas atividades e trazem enorme facilidade e praticidade às nossas vidas. Nesse sentido, o Apple II, desenvolvido pela Apple em 1977 foi um dos primeiros computadores pessoais a se popularizarem entre os consumidores. Mas não foi o primeiro.

Em 1971, surgia o primeiro computador pessoal da história: o Kenbak-1. Criado pela KENBA em 1971, o Kenbak-1 não teve muito sucesso comercial, tendo vendido apenas 40 unidades em 2 anos, sendo descontinuado em 1973.

Isso, todavia, não aconteceu com a Apple. Conforme aponta Kahney (2008), a ideia de Steve Jobs com a criação do Apple II não era, em um primeiro momento, a criação de computadores para uso pessoal, mas sim a atração de aficionados por tecnologia. Além disso, havia uma preocupação enorme com o design e com a completude do produto, de modo que a intenção era a entrega de um computador completo (com todos os periféricos), o que caracterizou o Apple II à época e algo que caracteriza os melhores computadores da atualidade: a possibilidade de substituição individual das peças. O Apple II foi um tremendo sucesso. Segundo informações de Kahney (2008), o Apple II foi lançado em 1977 e, em 1978, fez US\$ 700.000,00 em vendas, saltando para US\$ 7,9 milhões em 1979.

Mas o que diferenciou o Apple II do Kenbak-1? Certamente foi a diferenciação de produtos. Conforme apontado por Kahney (2008), a experiência com o Apple II trouxe como conhecimento à Apple que o design e a experiência do usuário com o produto eram essenciais para o seu sucesso comercial, de modo que essa noção se tornou uma das filosofias fundamentais da empresa. A Apple não inventou, no sentido que utilizamos neste artigo, a ideia de computador pessoal e não foi a primeira a desenvolver um mas, se vê em Hasenclever e Ferreira (2013), a inovação é a invenção que possui sucesso comercial, e certamente a Apple inovou no mercado de computadores pessoais. A criação do Apple II foi o primeiro grande sucesso da empresa norteamericana, e seu carro chefe por alguns anos.

A diferenciação de produtos, conforme aponta Losekann e Gutierrez (2013), pode possuir dois aspectos: vertical, onde há um aumento da utilidade marginal total dos consumidores, isto é, uma melhoria objetiva, ou horizontal, onde há uma nova percepção pelos indivíduos em relação ao produto, como design, facilidade de uso, estética, etc. Até o momento havíamos citado as melhorias horizontais do Apple II. Todavia, conforme aponta Kahney (2008), é preciso notar que o Apple II possuía uma melhoria vertical considerável: o VisiCalc, primeiro aplicativo de planilhas que permitia a visualização e a computação de dados consideráveis de forma automática. Em sua visão, esse foi o principal fator de sucesso do Apple II.

A figura 1 ilustra algumas diferenças importantes do Apple II em relação a maioria dos computadores anteriores. Em primeiro lugar, é preciso lembrar que o computador é basicamente um mecanismo de processamento de dados. No Kenbak-1, por exemplo, o primeiro computador pessoal lançado, não havia um periférico capaz de transmitir o resultado desse 
processamento. Ele carecia justamente de uma tela. Com a introdução do VisiCalc, seria necessário um dispositivo de visualização que facilitasse seu uso. A tela, como ilustrado na Figura 1, também foi um dos grandes diferenciais do Apple II.

Figura 1 - Apple II.

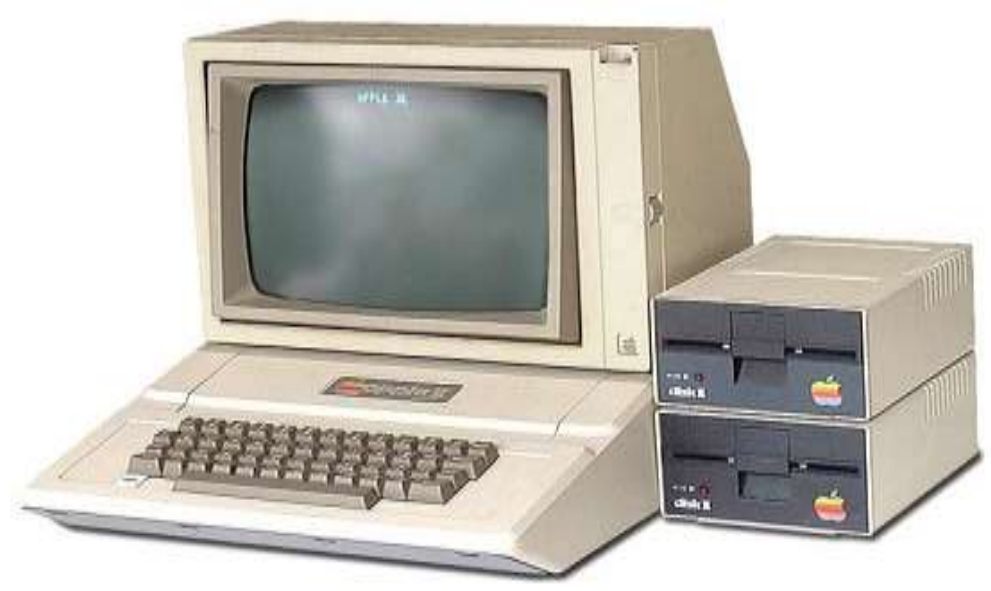

Fonte: Techtudo (2016).

\subsection{A Apple e o iPod}

Apesar do primeiro MP3 Player, denominado Rio PMP300 da empresa Diamond Multimedia, ter surgido em 1998, foi a partir do início dos anos 2000 que a sua popularidade aumentou entre os consumidores (Leão, 2015). Nesse sentido, identificouse que as necessidades desses consumidores se modificaram, tornando-se mais exigentes à medida em que players de música digital eram lançados e o design e capacidade de armazenamento já não eram tão satisfatórios, bem como a duração da bateria.

Conforme cita Kahney (2008), o projeto de elaboração do iPod contou com peças que já estavam disponíveis no mercado (bateria da Sony, chips de controle da Texas Instruments e o disco rígido fabricado pela Toshiba), bem como o uso do projeto básico de design que foi adquirido da PortalPlayer. E, apostando em um processo de diferenciação de produto, com melhoria em aspectos de design para que ele fosse compacto, bem como em capacidade de armazenamento e com a click wheel, que se tornava uma das características mais marcantes (Kahney, 2008), o iPod era lançado no mercado em 2001 com enorme sucesso comercial.

O produto passou por diversas atualizações, e melhorias ao longo dos anos, mas seu sucesso é indiscutível. Conforme menciona Kahney (2008), até abril de 2007 mais de 100 milhões de unidades foram vendidas e a expectativa era de que esse número fosse superado, o que se verificou posteriormente, pois, até o ano de 2010, a empresa havia vendido 275 milhões unidades do aparelho.

A Figura 2 mostra, de um lado, um dos primeiros MP3 players lançados no mercado, o Rio PMP300 e, do outro, o iPod. É possível notar através da imagem que há semelhanças nas funções exercidas pelos dois produtos, o que mostra que a ideia de MP3 player não foi introduzida no mercado pela Apple e que, do mesmo modo, ela não foi a primeira a lançar um produto desta espécie. No entanto, é perceptível o grau de diferenciação do produto, que possui um design mais sofisticado em relação aos outros produtos do período, atributo este que é um dos grandes responsáveis pelo sucesso do produto desde seu lançamento. 
Figura 2 - Comparação entre um dos primeiros MP3 players lançados, o Rio PMP300 à esquerda, e a primeira versão do iPod, à direita.

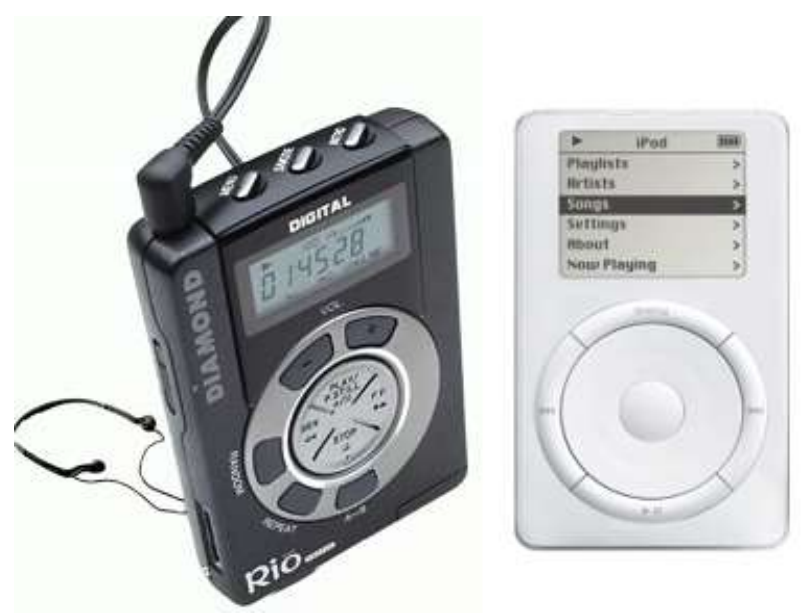

Fonte: Techtudo (2019) e Apple (2020).

É importante ressaltar que, durante muito tempo, o iPod liderou o setor no mercado. Apesar disso, conforme novas tecnologias eram lançadas no mercado, novas necessidades por parte dos consumidores surgiam. E, com a expansão do mercado de smartphones, a Apple lança, em 2007, o primeiro Iphone, um aparelho que possuía as funções de um celular comum, com ferramentas adicionais, mas combinado com os recursos de vídeo e áudio que o iPod disponibilizava.

Ao observar o Gráfico 1, é possível notar que a introdução do iPhone no mercado de aparelhos celulares gerou um retorno muito positivo para a Apple, que passou a investir no produto trazendo novas versões dele a cada ano, resultando em um aumento considerável no consolidado de vendas, principalmente a partir de 2010, bem como nos anos subsequentes. No entanto, também é possível verificar que, a partir desse mesmo período, o iPod apresenta uma queda acentuada no consolidado de vendas, ficando abaixo de 15 milhões de unidades vendidas em 2014, quando a empresa decide parar de divulgar os dados de vendas do produto em sua própria categoria, como aponta Vailshery (2021).

Gráfico 1 - Comparativo de vendas entre iPod e iPhone entre 2006 e 2014.

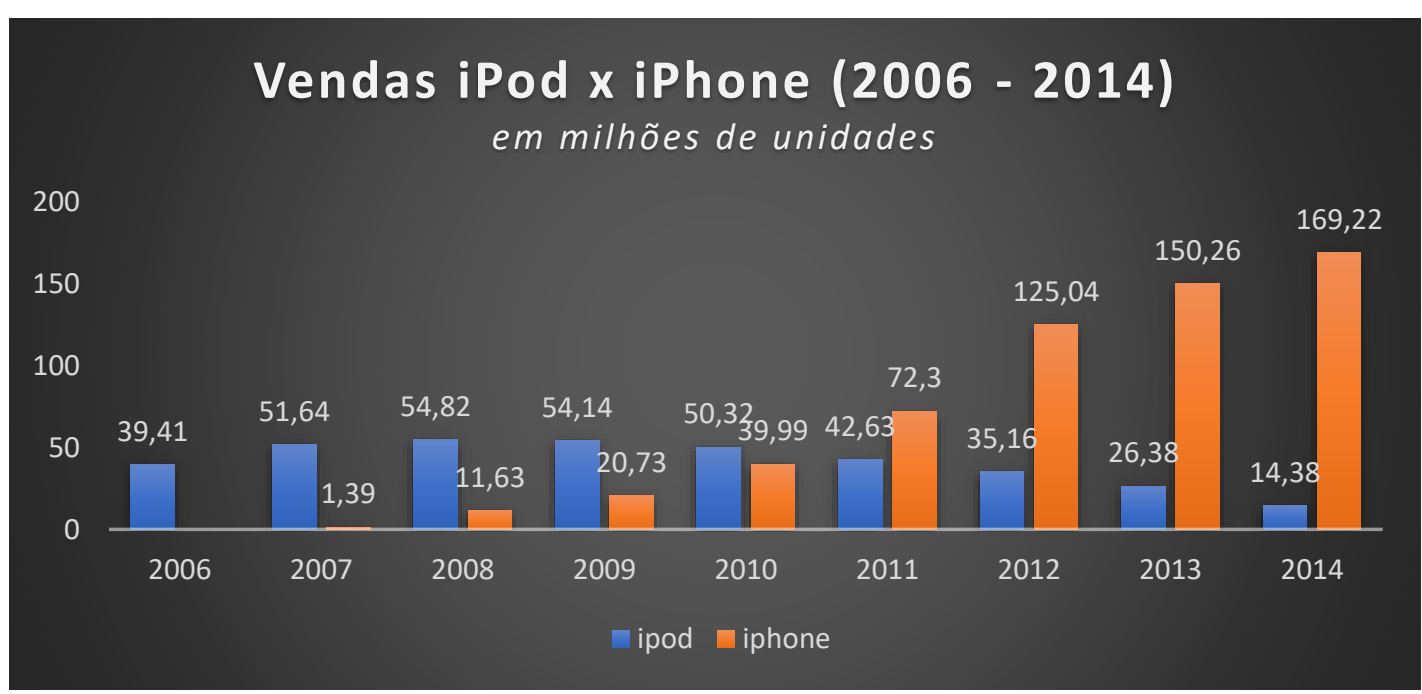

Fonte: Elaboração própria a partir de dados extraídos do Statista, com publicação de Vailshery, L. S. (2021). 
Esses dados mostram como a aposta em uma considerável diferenciação de produtos pode ser responsável por um crescimento notável. A estratégia adotada permite que a Apple não se limite ao espaço e nem aos preços dos produtos, de tal modo que, ainda que houvesse concorrência perfeita, seus produtos poderiam ser vendidos por um preço acima do estabelecido no mercado, possivelmente sem redução de receita.

\section{Conclusão}

Do exposto, pode-se concluir que a Apple adota uma estratégia de mercado, no que tange à questão da inovação, defensiva. Diferentemente de uma estratégia simplesmente imitativa, a Apple busca agregar tecnologias já existentes em produtos inovadores, visando a liderança de mercado. Dos casos analisados, não se pode dizer que em algum deles a ideia se tratou de uma invenção em sentido próprio, mas sim de inovação, isto é, a aplicação de invenções em processos comerciais de sucesso. A Apple também não buscou, como nos casos analisados, ser a primeira a lançar determinada tecnologia nova no mercado, mas busca uma imitação da tecnologia focando na diferenciação e melhoria dos produtos. Por um lado, no que se refere à inovação horizontal, trabalha sobremaneira com questões como design, experiência do usuário, percepção do produto, etc.. Por outro lado, sempre busca atribuir novas funcionalidades aos produtos que cria, visando uma inovação vertical considerável, o que aumenta a utilidade marginal total de seus consumidores e permite que seus produtos sejam vendidos a um preço consideravelmente mais elevado, principalmente em função da diferenciação horizontal.

Com sua política de diferenciação de produtos, e de imitação de invenções com melhoramentos e inovações verticais e horizontais, a Apple conseguiu assumir uma posição de liderança no mercado, mantendo-a até os dias de hoje. Sua estratégia e sua dedicação à diferenciação parecem constituir o cerne de seu sucesso comercial.

É importante ressaltar que algumas análises de empresas, do ponto de vista da diferenciação, realizadas anteriormente, como, por exemplo, o artigo "Inovação e estratégia de diferenciação da indústria de revestimentos cerâmicos: um estudo de caso sobre a empresa Portobello" (Nascimento \& Fontgalland, 2021), permitem compreender como a diferenciação de produtos é adotada não só no ramo da tecnologia, mas também em setores mais tradicionais.

Apesar disso, seria interessante que novos trabalhos analisassem a utilização de estratégias defensivas e de diferenciação de produtos em outros setores da economia, permitindo verificar a eficácia dessa estratégia nos mais diferentes ramos da atividade econômica, com vias a produzir uma teoria sobre a estratégia defensiva que permitisse auxiliar novas empresas em suas atividades.

\section{Referências}

Apple. (2021). Apple reports third quarter results. Apple website. https://www.apple.com/newsroom/2021/07/apple-reports-third-quarter-results/

Apple. (2020). Identify your iPod model. Apple support. https://support.apple.com/en-us/HT204217

Brookshear, J. G. (2013). Ciência da computação: uma visão abrangente. (11a ed). Ed. Bookman.

Freeman, C. \& Soete, L. (2008). A economia da inovação industrial. Ed. Unicamp.

Fontgalland, I. (2021) Slides MEC, UFCG.

Hasenclever, L. \& Ferreira, P. M. (2013). Estrutura de Mercado e Inovação. In: Kupfer, D. \& Hasenclever, L. (orgs.). Economia Industrial: fundamentos teóricos e práticas no Brasil. (2ed., cap. 8, p. 91-101). Ed. Elsevier.

Inácio, M. S. C. (2011). Como a estratégia seguida pela Apple se tornou um caso de sucesso. Dissertação de mestrado, Instituto Universitário de Lisboa, Lisboa, Portugal. https://repositorio.iscte-iul.pt/bitstream/10071/4077/1/APPLE_MIRIAM.pdf

Kahney, L. (2008). A cabeça de Steve Jobs. Ed. Agir.

Lakatos, E. M. \& Marconi, M. A. (2007). Fundamentos de metodologia científica. (8a ed). Ed. Atlas.

Leão, T. (2015). História do MP3: quem criou o formato musical? Mundo de músicas. https://mundodemusicas.com/historia-do-mp3/ 
Losenkann, L. \& Gutierrez, M. (2013). Diferenciação de Produtos. In: Kupfer, D. \& Hasenclever, L. (orgs.). Economia Industrial: fundamentos teóricos e práticas no Brasil. (2 ${ }^{\mathrm{a}}$ ed., cap. 6, p. 67-77). Ed. Elsevier.

Murphy, A. et al. (2021). Global 2000: as maiores empresas de capital aberto do mundo em 2021. Forbes. https://forbes.com.br/forbes-money/2021/05/global2000-as-maiores-empresas-de-capital-aberto-do-mundo-em-2021/

Nascimento, L. J. de S. \& Fontgalland, I. L. (2021). Inovação e estratégia de diferenciação da indústria de revestimentos cerâmicos: um estudo de caso sobre a empresa Portobello. E-Acadêmica, 2(3), e292358. https://doi.org/10.52076/eacad-v2i3.58

Nishikiori, I. (2019). 'Primeiro' MP3 player à venda faz 21 anos: veja curiosidades do Rio PMP300. Techtudo. https://www.techtudo.com.br/listas/2019/09/primeiro-mp3-player-a-venda-faz-21-anos-veja-curiosidades-do-rio-pmp300.ghtml

Nuttall, C. (2011). Aos 10 anos, iPod é líder do setor, mas vendas declinam. Valor Econômico. https://valor.globo.com/empresas/noticia/2011/11/04/aos-10anos-ipod-e-lider-do-setor-mas-vendas-declinam.ghtml

Sacramento, G. M. (2011). A estratégia da inovação por diferenciação de produtos: estudo de caso da Apple. Trabalho de conclusão de curso de graduação, Universidade Federal do Rio de Janeiro, Rio de Janeiro, RJ, Brasil. https://pantheon.ufrj.br/bitstream/11422/2217/1/GMSacramento.pdf

Segall, K. (2017). Incrivelmente simples: a obsessão que levou a Apple ao sucesso. Ed. Alta Books.

Souza, E. (2016). Apple faz 40 anos; relembre lançamentos das últimas quatro décadas. Techtudo. https://www.techtudo.com.br/listas/noticia/2016/04/applefaz-40-anos-relembre-lancamentos-das-ultimas-quatro-decadas.html.

Statista Research Department. (2014). Global Apple Ipode sales from 2006 to 2014. Statista. https://www.statista.com/statistics/276307/global-apple-ipod-salessince-fiscal-year-2006/

UOL. (2010). Com 275 milhões de iPod vendidos, Apple apresenta nova linha do tocador musical. UOL Notícias. Tecnologia. https://tecnologia.uol.com.br/mundo-apple/ultimas-noticias/2010/09/01/apple.jhtm

Vailshery, L. S. (2021). iPhone, iPad and iPod sales from 1st quarter 2006 to 4th quarter 2018. Statista. https://www.statista.com/statistics/253725/iphone-ipadand-ipod-sales-comparison/

Vaz, J. V. (2020). Administração estratégica - um estudo de caso baseado na Apple. Research, Society and Development, 9(9), e335997137. https://doi.org/10.33448/rsd-v9i9.7137

Yin, R. K. (2001). Estudo de caso: planejamento e métodos. (2a ed.). Ed. Bookman.

Zuckerman, A. \& Ive, J. (2016). Designed by Apple in California. Apple Inc. 